\title{
The new World Health Organization classification of lung tumours
}

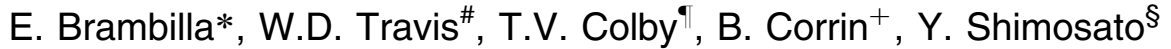

\begin{abstract}
The new World Health Organization classification of lung tumours. E. Brambilla, W.D. Travis, T.V. Colby, B. Corrin, Y. Shimosato. C ERS Journals Ltd 2001.

ABSTRACT: Tumour classification systems provide the foundation for tumour diagnosis and patient therapy and a critical basis for epidemiological and clinical studies. This updated classification was developed with the aim to adhere to the principles of reproducibility, clinical significance, and simplicity in order to minimize the number of unclassifiable lesions.

Major changes in the revised classification as compared to the previous one (WHO 1981 [1]) include the addition of two pre-invasive lesions to squamous dysplasia and carcinoma in situ; atypical adenomatous hyperplasia and diffuse idiopathic pulmonary neuroendocrine cell hyperplasia. Another change is the subclassification of adenocarcinoma: the definition of bronchioalveolar carcinoma has been restricted to noninvasive tumours. There has been substantial evolution of concepts in neuroendocrine lung tumour classification. Large cell neuroendocrine carcinoma (LCNEC) is now recognized as a histologically high grade non small cell carcinoma showing histopathological features of neuroendocrine differentiation as well as immunohistochemical neuroendocrine markers. The large cell carcinoma class has been enriched with several variants, including the LCNEC and the basaloid carcinoma, both with a dismal prognosis. Finally, a new class was defined called carcinoma with pleomorphic, sarcomatoid, or sarcomatous elements, which brings together a number of proliferations characterized by a spectrum of epithelial to mesenchymal differentiation.

Immunohistochemistry and electron microscopy are invaluable techniques for diagnosis and subclassification, but our intention was to render the classification simple and practical to every surgical laboratory, so that most lung tumours could be classified by light microscopic criteria.
\end{abstract}

Eur Respir J 2001; 18: 1059-1068.

\begin{abstract}
*Service de Pathologie Cellulaire, Centre Hospitalier Universitaire de Grenoble, Grenoble, France. " Dept of Pulmonary and Mediastinal Pathology, Armed Forces Institute of Pathology, Washington, USA. "Dept of Pathology, Mayo Clinic, Scottsdale, USA. 'Dept of Histopathology, Royal Brompton Hospital, National Heart and Lung Hospital, London, UK. ${ }^{\$} \mathrm{Cli}-$ nical Laboratory Division, National Cancer Center Hospital, Tokyo, Japan.
\end{abstract}

Correspondence: E. Brambilla Service de Pathologie Cellulaire Centre Hospitalier Universitaire de Grenoble

B.P. 217-38043 Grenoble Cedex France

Fax: 33476765949

Keywords: Histological classification lung tumours

World Health Organization

Received: July 212001

Accepted: July 222001
Lung cancer is currently the most frequently diagnosed major cancer and the most common cause of cancer mortality in males worldwide. This is largely due to the effects of cigarette smoke. It has been 17 years since the World Health Organization (WHO) classification was updated in 1981 [1], and since this time considerable progress has been made in understanding the epidemiological histogenetic and molecular biology of lung cancer. In 1994, the WHO adopted the International Association for the Study of Lung Cancer (IASLC) pathology panel to comprise the core membership of the WHO committee, to develop a new revised classification of lung and pleural tumours. Because of the contribution of IASLC in this project, this classification is called the WHO/IASLC Histological Classification of Lung and Pleural Tumours [2] (table 1). An international system of tumour classification is important for consistency in patient treatments and to provide the basis of epidemiological and biological studies. In developing this classification, pathologists have tried to adhere to the principles of reproducibility, clinical significance and simplicity, and to minimize the number of unclassifiable lesions. Most of this classification is based on the histological characteristics of tumours seen in surgical or needle biopsy, and is primarily based on light microscopy, although immunohistochemistry and electron microscopy findings are provided when necessary. Changes in classification were based essentially on the available published data or on the collective experience of the panel members when these date were not available. Certain timehonoured terms were retained, in order to respect their significance to the clinicians, while new concepts had

Previous articles in this series: No. 1: Steels E, Paesmans M, Berghmans T, et al. Role of p53 as a prognostic factor for survival in lung cancer: a systematic review of the literature with a meta-analysis. Eur Respir J 2001; 18: 705-719. No. 2: Van Klaveren RJ, Habbena JDF, Pedersen JH, de Koning HJ, Oudkerk M, Hoogsteden HC. Lung cancer screening by low-dose spiral computed tomography. Eur Respir $J$ 2001; 18: $857-866$. 
Table 1.-The 1999 World Health Organization/International Association for the Study of Lung Cancer Histological Classification of Lung and Pleural Tumours

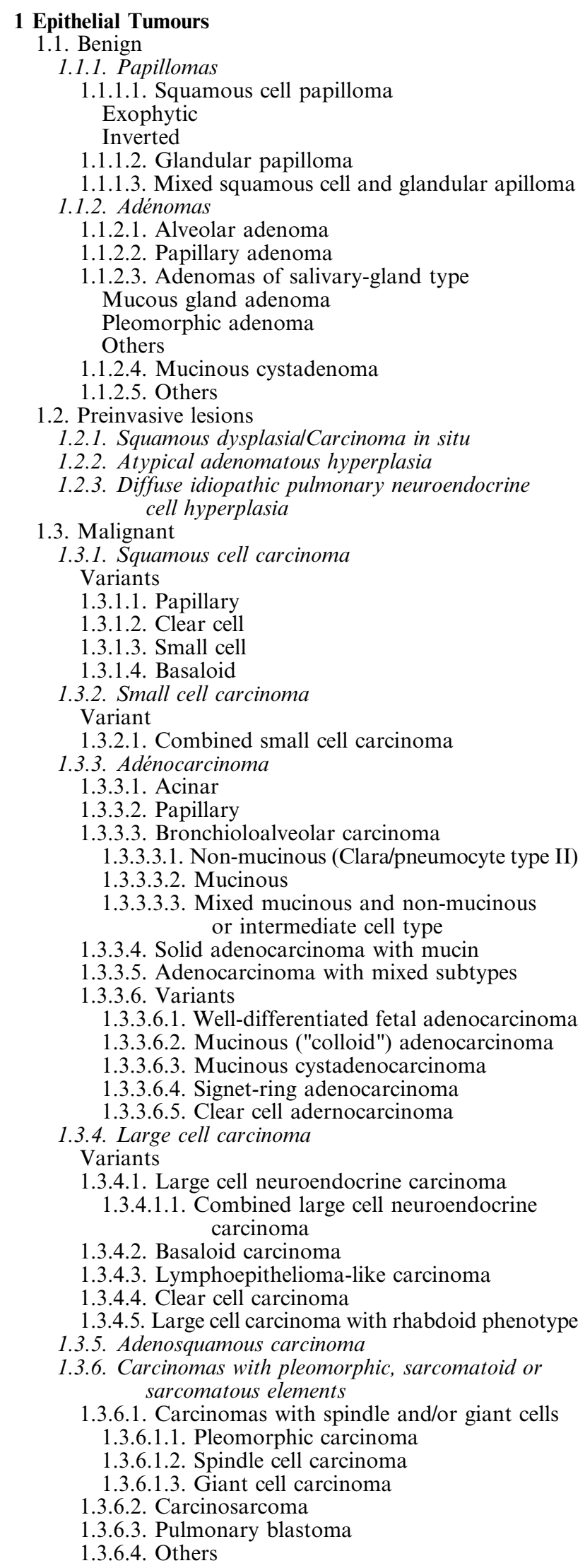

Table 1. Continued

1.3.7. Carcinoid tumour

1.3.7.1. Typical carcinoid

1.3.7.2. Atypical carcinoid

1.3.8. Carcinomas of salivary-gland type

1.3.8.1. Mucoepidermoid carcinoma

1.3.8.2. Adenoid cystic carcinoma

1.3.8.3. Others

1.3.9. Unclassified carcinoma

2 Soft Tissue Tumours

2.1 Localized fibrous tumour

2.2 Epithelioid hemangioendothelioma

2.3 Pleuropulmonary blastoma

2.4 Chondroma

2.5 Calcifying fibrous pseudotumour of the pleura

2.6 Congenital peribronchial myofibroblastic tumour

2.7 Diffuse pulmonary lymphangiomatosis

2.8 Desmoplastic small round cell tumour

2.9 Other

3 Mesothelial Tumours

3.1 Benign

3.1.1 Adenomatoid tumour

3.2 Malignant

3.2.1 Epithelioid mesothelioma

3.2.2 Sarcomatoid mesothelioma

3.2.2.1 Desmoplastic mesothelioma

3.2.3 Biphasic mesothelioma

3.2.4 Other

4 Miscellaneous Tumours

4.1 Hamartoma

4.2 Sclerosing hemangioma

4.3 Clear cell tumour

4.4 Germ cell neoplasms

4.4.1 Teratoma, mature or immature

4.4.2 Malignant germ cell tumour

4.5 Thymona

4.6 Melanoma

4.7 Others

5 Lymphoproliferative Disease

5.1 Lymphoid interstitial pneumonia

5.2 Nodular lymphoid hyperplasia

5.3 Low-grade marginal zone B-cell lymphoma of the mucosa-associated lymphoid tissue

5.4 Lymphomatoid granulomatosis

6 Secondary Tumours

7 Unclassified Tumours

8 Tumour-like Lesions

8.1 Tumourlet

8.2 Multiple meningothelioid nodules

8.3 Langerhans cell histiocytosis

8.4 Inflammatory pseudotumour (Inflammatory myofibroblastic tumour)

8.5 Organizing pneumonia

8.6 Amyloid tumour

8.7 Hyalinizing granuloma

8.8 Lymphangioleiomyomatosis

8.9 Multifocal micronodular pneumocyte hyperplasia

8.10 Endometriosis

8.11 Bronchial inflammatory polyp

8.12 Others

emerged that would necessitate a change. An example of this is "small-cell lung carcinoma". As compared to the previous edition, changes include a better definition of pre-invasive lesion, a reclassification of adenocarcinoma, the description of two new tumour types as variants of large cell carcinoma, large cell neuroendocrine carcinoma (LCNEC) and basaloid 
carcinoma, and a new class called pleomorphic carcinoma.

\section{Pre-invasive lesions}

More precise guidelines for grading squamous dysplasia in mild, moderate and severe dysplasia and to define carcinoma in situ are provided (fig. 1a-d). These precise criteria allowed better interobserver reproductibility [3]. In addition of the categories of squamous dysplasia and carcinoma in situ, two additional lesions were added to the group of preinvasive lesions: atypical adenomatous hyperplasia (AAH) and diffuse idiopathic pulmonary neuroendocrine cell hyperplasia (DIPNECH).

\section{Atypical adenomatous hyperplasia}

Many studies now justify that AAH is a precursor to adenocarcinoma [4-14]. Multiple synonyms have been used for this lesion including alveolar cell hyperplasia, atypical alveolar hyperplasia, bronchioloalveolar cell adenoma, alveolar atypical hyperplasia, and atypical alveolar cuboidal cell hyperplasia. $\mathrm{AAH}$ and nonmucinous bronchioloalveolar carcinoma (BAC) are lesions located most exclusively in lung periphery and a spectrum of neoplasia exists in an AAH-BAC sequence. However, most studies have examined these lesions as incidental findings in lung specimens resected for lung cancer and few biological studies have investigated whether this lesion represents a precursor to adenocarcinoma. However, the concept that $\mathrm{AAH}$ is a precursor to invasive carcinoma is mainly based on the fact that they are primarily seen adjacent to adenocarcinoma and due to the fact that some are identified within the same mass in emergence with the adenocarcinoma.

Although extensive research has been made on autopsy series and the findings depend on the number and intensity of this search, it is clear that AAH are not present in each patient developing an adenocarcinoma, and their frequency goes from $2 \%$ in young people with no cancer [15] to $23.2 \%$ in autopsy series of aged people with cancer [16]. Although it was tempting to establish a morphological grading of dysplasia along the spectrum of $\mathrm{AAH}$ and to separate lesions into low and high grade, pathologists at the WHO/IASLC panel did not find sufficiently convincing criteria to recommend grading. AAH is defined as a proliferation of minimally atypical cuboidal type II pneumonocytes with gaps between cells; AAH generally measures $<5 \mathrm{~mm}$ in diameter (fig. 1e, 1f). In addition to the morphology, additional data supporting the preneoplastic nature of $\mathrm{AAH}$ include comparative analysis of $\mathrm{AAH}$ and $\mathrm{BAC}$ showing progressive increase of a mean nuclear area by morphometry, alteration of proliferation measured by $\mathrm{Ki}-67$, progressive p53 accumulation and loss of heterozygosity at $3 p, 9 p$ and $17 \mathrm{p}$ and $\mathrm{K}$ (ras mutations at codon 12) in AAH and associated BAC [5, 8, 9, 13, 17-19].

\section{Diffuse idiopathic neuroendocrine cell hyperplasia}

Diffuse idiopathic neuroendocrine cell hyperplasia (DIPNECH) is a very rare disorder considered a precursor of tumourlets and typical or atypical carcinoids [20] (fig. 1g, 1h). Neuroendocrine cell hyperplasia occurs most commonly as a reactive hyperplasia in the setting of airway fibrosis and/or inflammation [21-22]. Two thirds of patients with peripheral carcinoid tumours have neuroendocrine cell hyperplasia in the adjacent bronchiolar epithelium. A subset of patients with DIPNECH has associated obstructive bronchiolar fibrosis with obstructive pulmonary functions. Some patients with DIPNECH have both obstructive lung disease and multiple tumourlets/ carcinoid tumours. Cytokines and growth factors secreted by neuroendocrine cells (gastrin-releasing peptide, fibroblast growth factors) suggest that these neuroendocrine cells are the cause of fibrosis [21]. A number of observations associating neuroendocrine cell hyperplasia and carcinoids suggests that this condition is a preneoplastic state for peripheral carcinoid tumours.

\section{Adenocarcinomas}

Adenocarcinoma is the predominant histological subtype of lung carcinoma in many countries [23-24]. In Europe, the incidence has increased by $10 \%$ in the last 20 yrs [25]. Adenocarcinoma is the most prevalent form of lung cancer in younger males ( $<50$ yrs old) and in women of all ages, in never smokers, and in former smokers [26]. Differences in tobacco smoking habits (filters, light tobacco cigarettes, profound inhalation) appear to favour the development of distal bronchiolar and alveolar carcinogenesis at the expense of proximal squamous cell carcinoma [27, 28].

Difficulties in adenocarcinoma subclassification arise from the fact that adenocarcinomas are highly heterogeneous histologically, with only a minority of cases showing a pure histological pattern. Mixed pattern adenocarcinomas are more common than tumours showing a single pattern (e.g. acinar, papillary, bronchioloalveolar, and solid adenocarcinoma with mucin formation).

The 1999 WHO classification is more restrictive in the definition of BAC; this category includes only noninvasive tumours with lepidic spread (fig. 2a, 2b). This present definition excludes cases with stromal, vascular or pleural invasion. Tumours showing these features are classified as adenocarcinoma mixed type with predominant bronchioloalveolar pattern (fig. 2c, 2d). Most tumours described as BAC in the past would now be in this category. The present definition of BAC is much more restrictive than any previously used by many pathologists. Since it has been shown that patients with solitary, noninvasive BACs measuring $<2.0 \mathrm{~cm}$ can be cured with resection [11], the current definition has a high clinical significance. Where BAC of Clara type II cells (nonmucinous) are usually solitary nodules and have very good prognosis, mucinous-type bronchioloalveolar carcinomas tend to spread and form satellite tumours or 

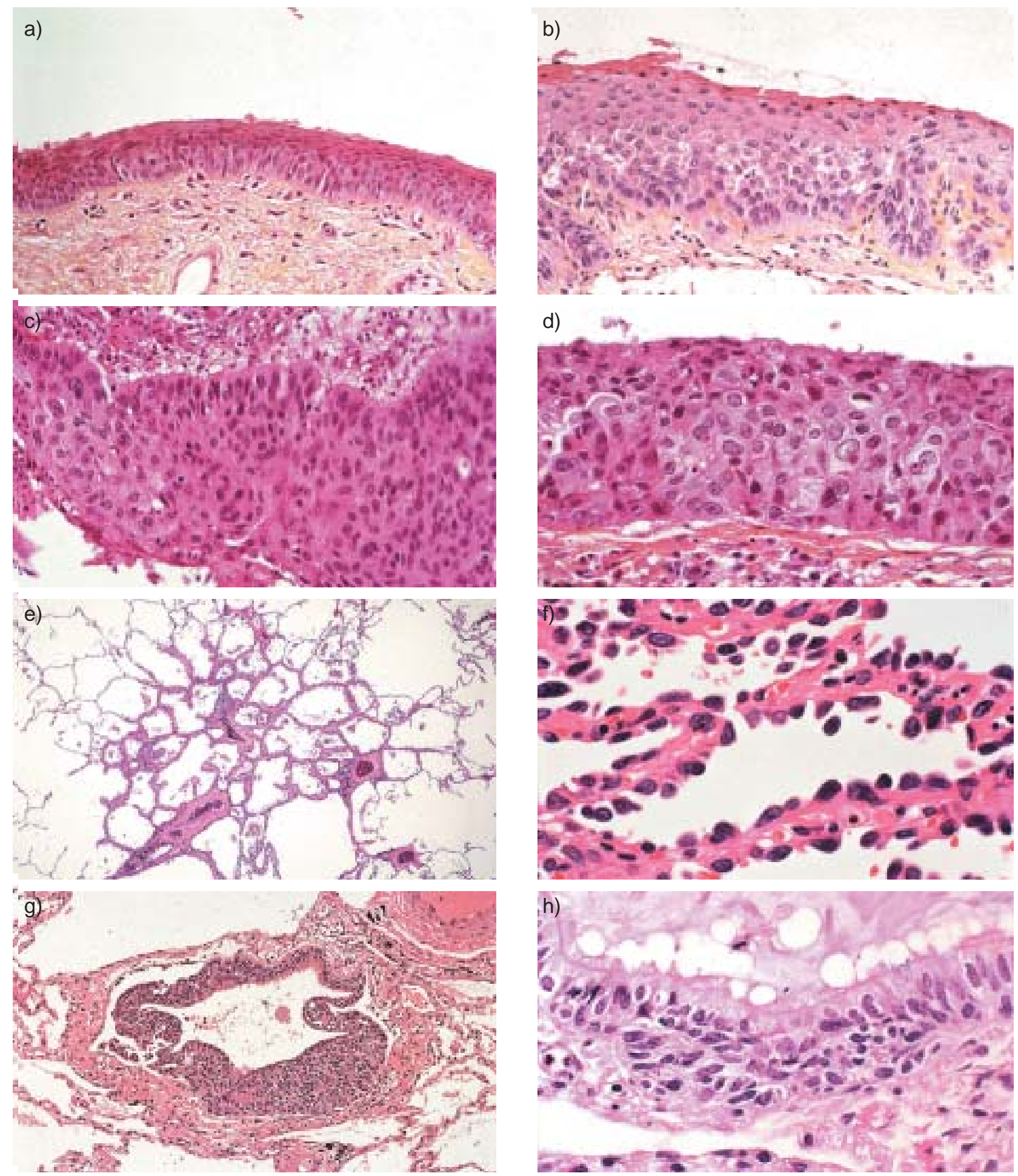

Fig. 1.- Histological patterns of significant and new entities. a) Mild dysplasia: mildly increased thickness, mild pleiomorphism, basilar zone expanded with cellular crowding in lower one-third of the epithelium, with nuclei vertically oriented, mitoses absent. b) Moderate dysplasia: moderately increased thickness, moderate anysocytosis and pleomorphism; basilar zone expanded with cellular crowding in lower two-thirds of epithelium with nuclei vertically oriented, mitotic figures in lower third. c) Severe dysplasia: markedly increased thickness, marked anisocytosis and pleomorphism, basilar zone expanded with cellular according into upper third, little progression of maturation, irregular nuclei vertically oriented in lower third, mitosis in lower two-thirds. d) Carcinoma in situ: markedly increased cell size and pleomorphism, no progression of maturation, cellular crowding throughout epithelium, no consistent orientation of nuclei, irregular nuclei with coarse, uneven chromatin, mitotic figures through full thickness. e) Atypical alveolar hyperplasia: a small millimetre-sized $(<5 \mathrm{~mm})$ bronchioloalveolar lesion consisting of proliferative pneumonocytes in a ledipic fashion along thin alveolar walls. f) Atypical alveolar hyperplasia: at high magnification, cuboidal type II pneumonocytes line the alveolar walls with gaps between adjacent cells (reproduced from World Health Organization (WHO) [2]). g) Diffuse idiopathic pulmonary neuroendocrine cell hyperplasia: the bronchioloalveolar epithelium is almost replaced by proliferating neuroendocrine cells (reproduced from WHO [2]). h) Diffuse idiopathic pulmonary neuroendocrine cell hyperplasia: at higher magnification, hyperplastic neuroendocrine cells form a nest at the base of the bronchiolar epithelium (reproduced from WHO [2]). 

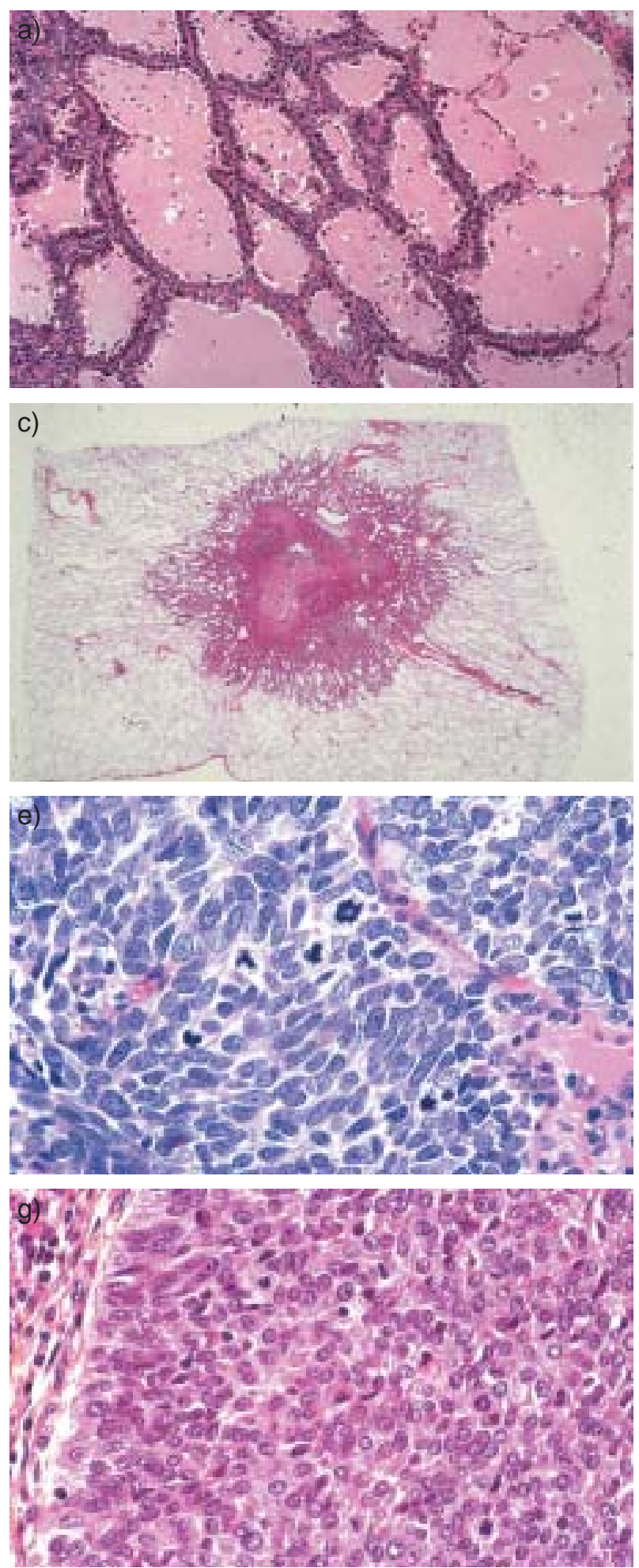
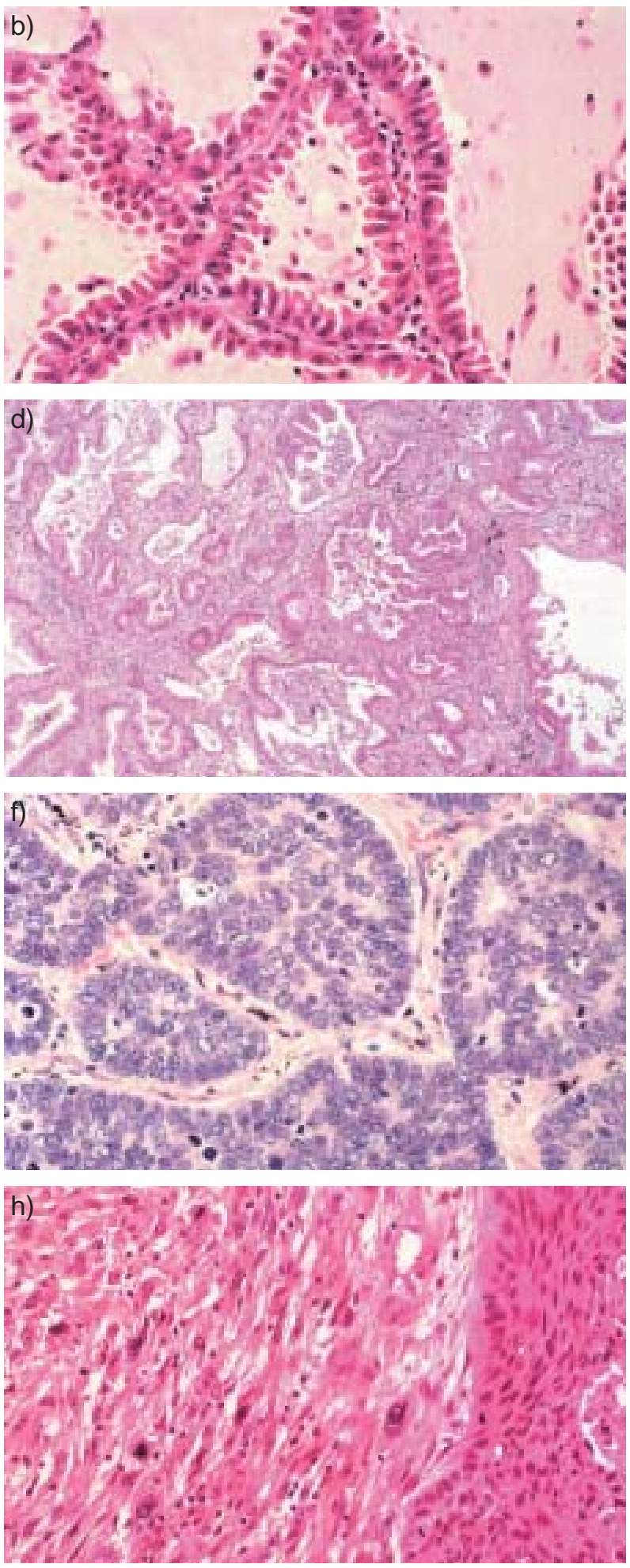

Fig. 2. - Histological patterns of significant and new entities. a) Bronchioloalveolar carcinoma, nonmucinous: the cuboidal to columnar shaped cells grow in a lepidic pattern along proexisting alveolar wall (reproduced from World Health Organization (WHO) [2]). b) Bronchioloalveolar carcinoma: cuboidal- to columnar-shaped cells (Clara cell differentiation) have basally oriented nuclei and eosinophilic cytoplasm with apical snouts (reproduced from WHO [2]). c) Adenocarcinoma with mixed subtypes: low magnification of an adenocarcinoma with a predominant bronchioloalveolar pattern that has an invasive component in a central scar. d) Adenocarcinoma with mixed subtypes: at higher magnification of the same case (c) the central scar contains an invasive acinar component. e) Small cell carcinoma: small tumour cells are densely packed, with scant cytoplasm, finely granular nuclear chromatin and absence of nucleoli. Mitoses are frequent (reproduced from WHO [2]). f) Large cell neuroendocrine carcinoma: palissading and rosette-like formation, numerous mitoses, vesicular nuclear chromatin (reproduced from WHO [2]). g) Basaloid carcinoma: palisading is conspicious at the periphery of the nests of tumour cells. Cells are hyperchromatic with relatively scant cytoplasm and show numerous mitotic figures. h) Pleomorphic carcinoma: this tumour has a mixture of spindle and giant cell carcinoma (left) with squamous cell component (right). 
pneumonic consolidation, and thus have a worse prognosis. AAH is considered a precursor for non mucinous BAC; there is no defined pre-invasive lesion for mucinous BAC.

A recent evaluation of surgically resected $\mathrm{BAC}$ detected in asymptomatic patients using systematic spiral computed tomography (CT) radiographic detection showed a high frequency of additional concomitant BACs, or AAH in $43 \%$ of patients [29]. It is expected that the presence of tiny multiple lesions (noninvasive and $<5 \mathrm{~mm}$ ) does not impact on prognosis [4].

Several variants of adenocarcinoma are recognized in the new WHO/IASLC classification including well-differentiated foetal adenocarcinoma, colloid carcinoma, mucinous cystadenocarcinoma, signet ring adenocarcinoma and clear cell adenocarcinoma. Since this classification work, thyroid transcription factor 1 (TTF-1) has appeared of great help in this differential diagnosis being expressed in $85 \%$ of primary lung adenocarcinoma and negative metastatic adenocarcinoma, although mucine producing primary adenocarcinoma might be TTF-1 negative.

\section{Large cell carcinoma and neuroendocrine tumours}

Large cell carcinoma now includes five variants in addition to large cell carcinoma defined as undifferentiated malignant epithelial cells. Two variants are recognized, both with dismal prognosis, LCNEC and basaloid carcinoma.

The concept of neuroendocrine tumours has been examined as a spectrum extending from low grade typical carcinoid to intermediate grade atypical carcinoid to high grade neuroendocrine tumours including large cell neuroendocrine carcinoma and small cell lung carcinoma (SCLC). Because of differences in clinical behaviour, therapeutic implications, and epidemiological context, these tumours have been presented separately in the WHO revised classification.

Typical and atypical carcinoid have been more precisely defined and clearly differentiated from each other with more objective criteria. Typical carcinoids have $<2$ mitotic figures per $2 \mathrm{~mm}^{2}$, and have no necrosis. The presence of necrosis, or a mitotic count between $2-10 \cdot 2 \mathrm{~mm}^{-2}$, allows separation of atypical carcinoid from typical carcinoid. A mitotic count $>10.2 \mathrm{~mm}^{-2}$ is seen in LCNEC. Using only very objective criteria (mitosis and necrosis), there is substantial interobserver reproducibility for subclassification of pulmonary neuroendocrine tumours; the most common disagreement involves LCNEC versus SCLC, followed by typical carcinoids versus atypical carcinoids. Subclassification of neuroendocrine tumours has been shown to be of strong prognostic implication on survival, allowing high significantly different survival rate between typical and atypical carcinoids $(\mathrm{p}<0.0001)$, and between atypical carcinoids and LCNEC $(p<0.0001)$. There is no significant survival differential rate between LCNEC and SCLC when stratified by stage [30-31]. LCNEC may be pure or combined, the latter if at least $10 \%$ of the tumours presents another nonsmall cell histology.
The diagnosis of LCNEC is based on recognition of both neuroendocrine morphology (organoid pattern) and the immunohistochemical demonstration of at least one specific neuroendocrine marker (fig. 2f). Among these specific neuroendocrine markers are chromogranin, synaptophysin and neural cell adhesion molecule (NCAM).

Neuroendocrine differentiation has been found immunohistochemically or ultrastructurally in $5-10 \%$ of NSCLC that do not display any neuroendocrine (organoid) morphology. The presence of neuroendocrine markers without neuroendocrine morphology does not allow a specific classification and is collectively referred to as "NSCLC with neuroendocrine differentiation" [32-35]. There is controversy over whether these tumours are associated with a worse or a better survival rate. Their clinical and therapeutic significance is not firmly established.

Although, IASLC had proposed to recognize a variant form of SCLC called mixed small cell-large cell carcinoma [36], this variant was not retained in revised WHO classification. Instead, SCLC is now described with only one variant: "SCLC combined", when at least $10 \%$ of the tumour bulk is made of an associated nonsmall cell component. SCLC presents a proliferation of small cells $(<4$ lymphocytes in diameter) with unique and strict morphological features, scant cytoplasm, ill-defined borders, finely granular "salt and pepper" chromatin, absent or inconspicuous nucleoli, frequent nuclear molding, and a high mitotic count (fig. 2e). The category of combined small cell carcinoma includes cases with a mixture of small cell and large cell or any other nonsmall cell component. Importantly for the clinical significance of the diagnostic signature, any cases showing at least $10 \%$ of SCLC is diagnosed as SCLC combined, even if the tumour has a heterogeneous sarcomatous component. SCLC alone is reserved to tumours with pure SCLC histology. SCLC associated with LCNEC is diagnosed as SCLC combined with LCNEC.

In addition, LCNEC comprise rare tumour types such as lymphoepithelioma-like carcinoma (EpsteinBarr virus-dependant epithelial proliferation) [37, 38], clear cell carcinoma [39], and large cell carcinoma with rhabdoid phenotype [40]. Large cell carcinoma with rhabdoid phenotype as well as blastoma and desmoid round cell tumours, primitive neuroendocrine tumour (PNET) and paraganglioma belong to the category of non-neuroendocrine tumours that may display neuroendocrine markers.

\section{Basaloid carcinoma}

Basaloid carcinoma is now defined as a variant of large cell carcinoma [41, 42]. Basaloid carcinoma presents with relatively small cells forming lobular pattern with a high rate of mitosis $\left(15-100 \cdot 2 \mathrm{~mm}^{-2}\right)$, peripheral palisading and comedo-type necrosis (fig. $2 \mathrm{~g}$ ). They represent $3 \%$ of nonsmall cell lung carcinoma (NSCLC) in Europe. In their pure type, they should not be confused with poorly differentiated squamous cell carcinoma, because they bear 
significantly poorer prognosis at stage I-II [42]. Thirty per cent of basaloid carcinoma disclose rosettes, but they are distinguished from LCNEC on the basis of tumour cell size, nuclear features and absence of immunohistochemical neuroendocrine markers. Like other NSCLC, basaloid carcinoma may display one or two neuroendocrine histochemical markers in a small proportion $(<10 \%)$ of the tumour cells. A specific set of cytokeratins (CK 1, 5, 10, and 14 recognized by the $34 \beta \mathrm{E} 12$ monoclonal antibody) is consistently absent in neuroendocrine tumours including LCNEC, and consistently expressed in basaloid carcinoma [43]. When a basaloid carcinoma shows squamous differentiation, the designation as basaloid variant of squamous cell carcinoma is appropriate (table 1). The basaloid variant of squamous cell carcinoma and pure basaloid carcinoma share the same dismal prognosis with a median survival time of 20 months [41, 44]. Interestingly basaloid carcinoma disclose a higher frequency of p53 mutation and p53 protein aberrant expression, a higher rate of $\mathrm{p} 16^{1 \mathrm{NK} 4}$ inactivation with loss of protein expression and higher Bcl2:bax ratio than squamous cell carcinoma and other NSCLC $[45,46]$.

\section{Carcinoma with pleomorphic, sarcomatoid, or sarcomatous elements}

These tumours are defined as a group of poorly differentiated nonsmall cell carcinoma that contains a component of sarcoma or sarcoma-like elements. They have been described under a variety of names including sarcomatoid carcinoma, spindle cell carcinoma, giant cell carcinoma, carcinosarcoma, blastoma and others. They are rare tumours and few pathologists have the opportunity to study very many of them. Spindle and giant cell carcinoma and carcinosarcoma comprise only $0.4 \%$ and $0.1 \%$ of all lung malignancies, respectively [23]. The tumours described under which name represent overall a continuum of epithelial and mesenchymal differentiation [47, 48]. Whether these tumours have a worse prognosis than other NSCLC is intuitively likely, but has not been firmly demonstrated on large series.

The great majority of carcinoma with components of spindle cells and/or giant cell carcinoma is histologically heterogeneous. Pleomorphic carcinoma shows carcinomatous as well as spindle and/or giant cell components (fig. 2h). Using antibodies against a large panel of cytokeratins, the epithelial nature of the spindle or giant cell component in these tumours can almost always be demonstrated. The epithelial markers might be negative on the spindle cell component, but an obvious carcinoma associated in the tumour allows the diagnosis of pleomorphic carcinoma in these cases. Pure spindle cell or giant cell carcinomas are extremely rare if the tumour has been widely sampled.

Carcinosarcoma is arbitrarily defined as a biphasic tumour with both carcinomatous and sarcomatous components, with the latter demonstrating heterologous elements such as malignant bone, cartilage, or skeletal muscle. It is controversial whether carcinosarcoma really exist. Most of the studies examining the presence of keratin in chondrosarcomatous or osteosarcomatous elements associated with epithelial components in these tumours have shown the presence of cytokeratin expression, favouring classification as a carcinoma with sarcomatoid elements, rather than carcinosarcoma. Consistent with this, molecular studies have demonstrated the same molecular and genetic abnormalities in both the epithelial and sarcomatoid components [49-53].

Pulmonary blastoma is considered as part of the spectrum of carcinomas with a pleomorphic sarcomatoid or sarcomatous element, based also on clinical pathological and molecular data. It is a biphasic tumour containing a primitive epithelial component resembling well-differentiated foetal-type adenocarcinoma and a primary mesenchymal stroma. Well-differentiated foetal adenocarcinoma is now included as an histological variant of adenocarcinoma and should not be regarded as a "monophasic" pulmonary blastoma, since the former has a much better prognosis and lacks p53 mutations in the latter. Pulmonary blastoma are observed in adults, whereas pleuropulmonary blastoma is a cystic and/or solid sarcoma (included in soft tissue tumours) arising in children $<6$ yrs old.

\section{Other tumours arising in the lung}

A number of tumours arising in the lung as the primary site are not described in details in the WHO classification of lung tumours because they have no specific features in the lung.

Carcinomas of salivary-gland type are described in detail in the WHO Histological Classification of Tumours of the Salivary Gland [54]. Mucoepidermoid carcinoma of high grade malignancy should be differentiated from adenosquamous carcinoma and from low grade mucoepidermoid carcinoma, which has a better prognosis. Adenoid cystic carcinomas predominantly arise in the trachea and large bronchi. Acinic cell carcinoma, epimyoepithelial carcinoma and malignant mixed tumours arising from bronchial glands are also described.

Soft tissue tumours are defined and classified in WHO Histological Typing of Soft Tissue Tumours [55]. Among these, some merit mention for their frequency in the lung: localized fibrous tumour (formerly called benign fibrous mesothelioma); epithelioid haemangioendothelioma (previously called intravascular bronchioloalveolar tumours); pleuropulmonary blastoma (which occurs in children and is distinguished from pulmonary blastoma which occurs in adults); chondroma (which mostly occurs in early childhood); calcifying fibrous pseudotumour of the visceral pleura); congenital peribronchial myofibroblastic tumours, diffuse pulmonary lymphangiomyomatosis (not to be confused with lymphangioleiomyomatosis) and; desmoplastic round cell tumour.

Lymphomas primary in the lung are mentioned briefly and the WHO Classification of leukaemia and lymphoma is now available for details [56]. Pleural 
mesothelial tumours are described briefly in WHO 1999 with no changes from previous classification [57].

\section{Role of immunohistochemistry and electron microscopy}

Immunohistochemistry and electron microscopy are indeed valuable techniques for diagnosis and subclassification of lung tumours; however, the authors have attempted to establish a classification that is simple, practical, and able to be used even in surgical pathology laboratories where these techniques are not available.

Nevertheless, immunohistochemistry and/or electron microscopy are required for diagnosis of LCNEC (specific neuroendocrine markers are chromogranin, synaptophysin, NCAM) and for the differential diagnosis between malignant mesothelioma and metastatic adenocarcinoma to the pleura (specific positive markers are calretinin and cytokeratin 5,6). These markers are necessary for the differential diagnosis between malignant mesothelioma and peripheral mesothelioma-like adenocarcinoma, but have been developed further to the WHO classification. The distinctive immunostaining characteristics of certain tumours are also very useful, such as HMB-45 positivity in clear cell tumours and lymphangioleiomyomatosis. Epidermic membrane antigen and TTF-1 immunostaining in sclerosing haemangiomas are of great help. In addition, TTF-1 has been shown to recognize specifically lung adenocarcinoma (85\% of the cases positive), whereas metastatic adenocarcinoma in the lung are always TTF-1 negative.

\section{Molecular studies}

The advent of molecular biology has also allowed a new depth of insights into the understanding of many lung tumours. For example, the molecular demonstration of the neoplastic nature of alveolar adenoma, chondroid hamartoma, and inflammatory pseudotumours (inflammatory myofibroblastic tumour) has been provided. Molecular and genetic abnormalities have highly contributed to the concept of bronchial or alveolar pre-invasive lesion as compared to invasive lung tumours.

\section{Conclusion}

Most lung tumours can be classified on the basis of light microscopy only, which provide a potential widespread and worldwide application of this classification system. Future studies should clarify some of the difficult problems in lung tumour classification, especially for pre-invasive lesions, adenocarcinoma subtyping, and carcinomas with pleomorphic, sarcomatoid, or sarcomatous elements.

Acknowledgements. The authors would like to thank W. Franklin (Denver, CO, USA) for its contribution on pre-invasive lesions; Chair,
International Association for the Study of Lung Cancer (IASLC)/National Cancer Institute SPORE Pathology Working group for the Classification of Pre-invasive Epithelial Abnormalities of Lung, the panel chairs and coordinators are also core panel members and IASLC pathology members, the extended panel of reviewers: S. Aisner ${ }^{\#}$ (Newark, NJ, USA), A. Churg (Vancouver, British Columbia, Canada), L.P. Dehner (St. Louis, MO, USA), A.F. Gazdar" (Dallas, TX, USA), D.W. Henderson (Bedford Park, South Australia), N.A. Jambhekar (Parel Bombay, India), M.N. Koss (Los Angeles, CA, USA), K.M. Müller (Bochum, Germany), N. Petrovitchev (Moscow, Russia), P. Saldiva (São Paulo, Brazil), M. Sheppard (London, UK), Sj.Sc. Wagenaar (Amsterdam, The Netherlands), W-h. Li (Beijing, P.R., China) ("IASLC panel members). The authors also thank J.M. Larsen and M. Lorinet for technical help.

Members of the WHO group. WHO Chairman: W.D. Travis; IASLC Chairman: F.R. Hirsch (Copenhagen, Denmark); Coordinators: T.V. Colby, B. Corrin, Y. Shimosato, E. Brambilla; Core Panel Members: E. AlvarezFernandez (Madrid, Spain), S.P. Hammar (Bremerton, WA, USA), P.S. Hasleton (Manchester, UK), B. Mackay (Houston, TX, USA), H. Popper (Graz, Austria), R.H. Steele (Woolloongabba, Australia).

\section{References}

1. World Health Organization. Histological Typing of Lung Tumours. 2nd Edn. Geneva, World Health Organization, 1981.

2. Travis WD, Colby TV, Corrin B, Shimosato Y, Brambilla E. In Collaboration with Sobin LH and Pathologists from 14 Countries. World Health Organization International Histological Classification of Tumours. Histological Typing of Lung and Pleural Tumours. 3rd Edn. Springer-Verlag, 1999.

3. Nicholson AG, Perry LJ, Cury PM, et al. Reproducibility of the WHO/IASLC grading system for preinvasive squamous lesions of the bronchus: a study of interobserver and intra-observer variation. Histopathology 2001; 38: 202-208.

4. Suzuki K, Nagai K, Yoshida J, et al. The prognosis of resected lung carcinoma associated with atypical adenomatous hyperplasia: a comparison of the prognosis of well-differentiated adenocarcinoma associated with atypical adenomatous hyperplasia and intrapulmonary metastasis. Cancer 1997; 79: 1521-1526.

5. Kitamura H, Kameda Y, Nakamura N, et al. Atypical adenomatous hyperplasia and broncholalveolar lung carcinoma. Analysis by morphometry and the expression of p53 and carcinoembryonic antigen. Am J Sug Pathol 1996; 20: 553-562.

6. Mori M, Tezuka F, Chiba R, et al. Atypical adenomatous hyperplasia and adenocacinoma of the human lung. Their heterology in form and analogy in immunohistochemical characteristics. Cancer 1996; 77: 665-674.

7. Miller RR. Bronchioloalveolar cell adenomas. Am J Surg Pathol 1990; 14: 904-912.

8. Kodama T, Biyajima S, Watanabe S, Shimosato Y. Morphometric study of adenocarcinomas and 
hyperplasitc epithelial lesions in the peripheral lung. Am J Clin Pathol 1986; 85: 146-151.

9. Kerr KM, Carey FA, King G, Lamb D. Atypical alveolar hyperplasia: relationship with pulmonary adenocarcinoma, p53, and c-erb-2 expression. $J$ Pathol 1994; 174: 249-256.

10. Kerr KM. Adenomatous hyperplasia and the origin of peripheral adenocarcinoma of the lung. In: Corrin B, ed. Pathology of Lung Tumours. London, Churchill Livingstone, 1997; pp. 119-134.

11. Noguchi M, Morikawa A, Kawasaki M, et al. Small adenocarcinoma of the lung. Histologic characteristics and prognosis. Cancer 1995; 75: 2844-2852.

12. Mori M, Chiba R, Takahashi T. Atypical adenomatous hyperplasia of the lung and its differentiation from adenocarcinoma. Cancer 1993; 72: 2331-2340.

13. Nakayama $\mathrm{H}$, Noguchi $\mathrm{M}$, Tsuchiya $\mathrm{R}$, et al. Clonal growth of atypical adenomatous hyperplasia of the lung: Cytofluometric analysis of nuclear DNA content. Mod Pathol 1990; 3: 214-320.

14. Kitamura H, Kameda Y, Ito T, Hayashi H. Atypical adenomatous hyperplasia of the lung. Implications for the pathogenesis of peripheral lung adenocarcinoma. Am J Clin Pathol 1999; 111: 610-622.

15. Sterner DJ, Mori M, Roggli VL, Fraire AE. Prevalence of pulmonary atypical alveolar cell hyperplasia in an autopsy population: a study of 100 cases. Mod Pathol 1997; 10: 469-473.

16. Yokose T. Clinicopathological analysis of atypical adenomatous hyperplasia-bronchioloalveolar carcinoma sequence of the lung. S731, Symposium on pre-invasive and invasive cancer of the lung. Nagoya, Japan, October, 2000

17. Kitaguchi $\mathrm{S}$, Takeshima $\mathrm{Y}$, Nishisaka $\mathrm{T}$, Inai $\mathrm{K}$. Proliferative activity, p53 expression and loss of heterozygosity on $3 p, 9 p$ and $17 p$ in atypical adenomatous hyperplasia of the lung. Hiroshima J Med Sci 1998; 47: 17-25.

18. Cooper CA, Carby FA, Bubb VJ, Lamb D, Kerr KM, Wyllie AH. The pattern of K-ras mutation in pulmonary adenocarcinoma defines a new pathway of tumour development in the human lung. J Pathol 1997; 181: 401-404.

19. Westra WH, Baas IO, Hruban RH, et al. K-ras oncogene activation in atypical alveolar hyperplasia of the human lung. Cancer Res 1996; 56: 2224-2228.

20. Aguayo SM, Miller YE, Waldron JA Jr, et al. Brief report: idiopathic diffuse hyperplasia of pulmonary neuroendocrine cells and airways disease. $N$ Engl J Med 1992; 327: 1285-1288.

21. Aguayo SM, King TE Jr, Waldron JA Jr, Sherritt KM, Kane MA, Miller YE. Increased pulmonary neuroendocrine cells with bombesin-like immunoreactivity in adult patients with eosinophilic granuloma. J Clin Invest 1990; 86: 838-844.

22. Gosney JR, Sissons MC, Allibone RO, Blakey AF. Pulmonary endocrine cells in chronic bronchitis and emphysema. J Pathol 1989; 157: 127-133.

23. Travis WD, Travis LB, Devesa SS. Lung cancer. Cancer 1995; 75: 191-202. (published erratum appears in Cancer 1995; 75: 2979.)

24. Takeshima Y, Nishisaka T, Kawano R, et al. p16/ CDKN2 gene and p53 gene alterations in Japanese non-smoking female lung adenocarcinoma. Jpn $J$ Cancer Res 1996; 87: 134-140.

25. Schottenfeld D. Etiology and epidemiology of lung cancer. In: Pass HI, Mitchell JB, Johnson DH, Turrisi
AT, Minna JD, eds. Lung Cancer: Principles and Practice. Philadelphia, USA, Lippincott Williams and Wilkins, 2000; pp. 367-388.

26. Ramalingam S, Pawlish K, Gadgeel S, Demers R, Kalemkerian GF. Lung cancer in young patients: analysis of a surveillance, epidemiology, and end results database. J Clin Oncol 1998; 16: 651-657.

27. Charloux A, Quoix E, Wolkove N, Small D, Pauli G, Kreisman $H$. The increasing incidence of lung adenocarcinoma: reality or artefact? A review of the epidemiology of adenocarcinoma. Int $J$ Epidemiol 1997; 26: 14-23.

28. Franceschi S, Bidoli E. The epidemiology of lung cancer. Ann Oncol 1999; 10: Suppl. 5, S3-S6.

29. Vasquez M, Flieder D, Yankelevitz D, Henschke C. Undetected pathologic lesions in lobectomy specimens of CT detected bronchioloalveolar carcinoma (BAC). Modern Pathol. Abstract 1341. Modern Pathol 2001; 14: 227A.

30. Travis MD, Linnoila RI, Tsokos MG, et al. Neuroendocrine tumors of the lung with proposed criteria for large-cell neuroendocrine carcinoma. An ultrastructural, immunohistochemical, and flow cytometric study of 35 cases. Am J Surg Pathol 1991; 15: 529553.

31. Travis WD, Rush W, Flieder DB, et al. Survival analysis of 200 pulmonary neuroendocrine tumors with clarification of criteria for atypical carcinoid and its separation from typical carcinoid. Am J Surg Pathol 1998; 22: 934-944.

32. Schleusener JT, Tazelaar HD, Jung SH, et al. Neuroendocrine differentiation is an independent prognostic factor in chemotherapy-treated non small cell lung carcinoma. Cancer 1996; 77: 1284-1291.

33. Linnoila RI, Piantadosi S, Ruckdeschel JC. Impact of neuroendocrine differentiation in non small cell lung cancer. The LCSG experience. Chest 1994; 106: 367S$371 \mathrm{~S}$.

34. Carles J, Rosell R, Ariza A, et al. Neuroendocrine differentiationas a prognostic factor in non small cell lung cancer. Lung Cancer 1993; 10: 209-219.

35. Neal MH, Kosinski R, Cohen P, Orenstein JM. Atypical endocrine tumors of the lung: a histologic, ultrastructural, and clinical study of 19 cases. Hum Pathol 1986; 17: 1264-1277.

36. Hirsch FR, Matthews MJ, Aisner S, et al. Histopathologic classification of small cell lung cancer. Changing concepts and terminology. Cancer 1988; 62: 973-977.

37. Butler AE, Colby TV, Weiss L, Lombard CM. Lymphoepithelioma-like carcinoma of the lung. Am J Surg Pathol 1989; 13: 632-639.

38. Chan JK, Hui PK, Tsang WY, et al. Primary lymphoepithelioma-like carcinoma of the lung. A clinicopathologic study of 11 cases. Cancer 1995; 76: 413-422.

39. Katzenstein AL, Prioleau PG, Askin FB. The histologic spectrum and significance of clear cell change in lung carcinoma. Cancer 1980; 45: 943-947.

40. Cavazza A, Colby TV, Tsokos M, Rush W, Travis WD. Lung tumors with a rhabdoid phenotype. Am J Clin Pathol 1996; 105: 182-188.

41. Brambilla E, Moro D, Veale D, et al. Basal cell (basaloid) carcinoma of the lung: A new morphologic and phenotypic entity with separate prognosis significance. Human Pathol 1992; 23: 993-1003.

42. Moro D, Brichon PY, Brambilla E, Veale D, Labat-Moleur F, Brambilla C. Basaloid bronchial 
carcinoma. A histological group with a poor prognosis. Cancer 1994; 73: 2734-2739

43. Sturm N, Lantuejoul S, Laverriere MH, et al. Thyroid transcription factor-1 (TTF-1) and cytokeratin 1, 5, 10, 14 (34betaE12) expression in basaloid and large cell neuroendocrine carcnomas of the lung. Hum Pathol 2001; 32: 918-925.

44. Brambilla E. Basaloid carcinoma. In: Brambilla C, Brambilla E, eds. Lung Tumors: Fundamental Biology and Clinical Management. New York and Basel, M. Dekker, 1998; pp. 13-28.

45. Brambilla E, Negoescu A, Gazzeri S, et al. Apoptosisrelated factors $\mathrm{P} 53, \mathrm{Bcl} 2$, and $\mathrm{Bax}$ in neuroendocrine lung tumors. Am J Pathol 1996; 149: 1941-1952.

46. Brambilla E, Moro D, Gazzeri S, Brambilla C. Alterations of $\mathrm{Rb}, \mathrm{P} 16^{\mathrm{INK} 4}$, Cyclin D1 expressions in non small cell lung carcinoma and their clinical significance. J Pathol 1999; 188: 351-360.

47. Fishback NF, Travis WD, Moran CA, Guinee DG Jr, McCarthy WF, Koss MN. Pleomorphic (spindle/giant cell) carcinoma of the lung. A clinicopathologic correlation of 78 cases. Cancer 1994; 73: 2936-2945.

48. Nappi O, Glasner SD, Swanson PE, Wick MR. Biphasic and monophasic sarcomatoid carcinomas of the lung. A reappraisal of "carcinosarcomas" and "spindle-cell carcinomas". Am J Clin Pathol 1994; 102: 331-340.

49. Matsui K, Kitagawa M, Miwa A. Lung carcinoma with spindle cell components: sixteen cases examined by immunohistochemistry. Hum Pathol 1992; 23: 1289-1297.
50. Addis BJ, Corrin B. Pulmonary blastoma, carcinosarcoma, and spindle-cell carcinoma: an immunohistochemical study of keratin intermediate filaments. J Pathol 1985; 147: 291-301.

51. Suster S, Huszar M, Herczeg E. Spindle-cell carcinoma of the lung immunocytochemical and ultrastructural study of a case. Histopathology 1987; 11: 871-878.

52. Wick MR, Swanson PE. "Carcinosarcomas" current perspectives and a histological review of nosological concepts. Semin Diagn Pathol 1993; 10: 118-127.

53. Thompson L, Chang B, Barsky SH. Monoclonal origins of malignant mixed tumors (carcinosarcomas). Am J Surg Pathol 1996; 20: 277-287.

54. Seifert G, ed. World Health Organization International Histological Classification of Tumours. Histological typing of salivary gland tumours. Springer-Verlag, 1991.

55. Weiss SW, ed. World Health Organization International Histological Classification of Tumours. Histological typing of soft tissue tumours. SpringerVerlag, 1994.

56. Jaffe ES, Harris NL, Stein H, Vardinan J, eds. WHO Classification of Tumours (IARC Edn.). Pathology and genetics of tumours of haematopoietic and lymphoid tissues. Lyon, IARC Press, 2001 (in press).

57. Battifora H, Elliot McCaughey WT. Atlas of Tumor Pathology: Tumors of the Serosal Membranes. 3rd Series: Fasicle 15. New York, Washington, Armed Forces Institute of Pathology, 1995. 